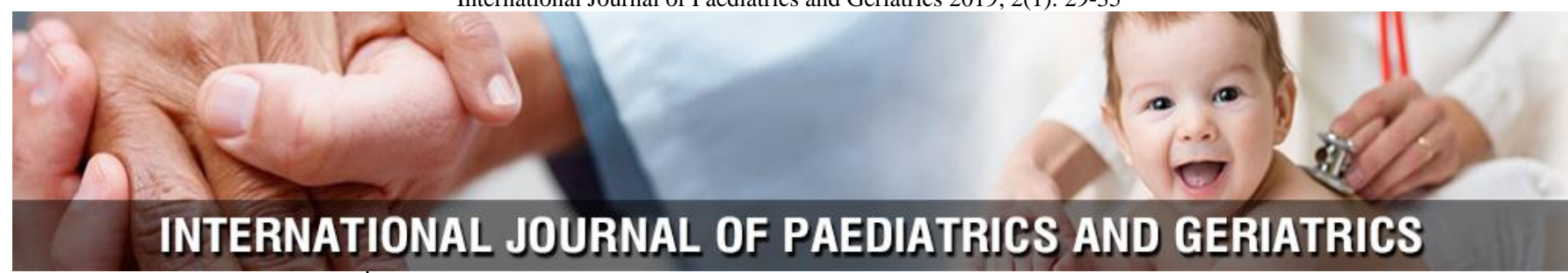

P-ISSN: 2664-3685

E-ISSN: 2664-3693

www.paediatricjournal.com

IJPG 2019; 2(1): 29-35

Received: 25-11-2018

Accepted: 28-12-2018

Dr. Basma Jasims Obaid

Central Teaching Hospital of

Pediatric, Baghdad, Iraq

Dr. Iman Abdul Wahhab

Hasan

Central Teaching Hospital of

Pediatric, Baghdad, Iraq

Dr. Sarab Shakir Abbas

Central Teaching Hospital of

Pediatric, Baghdad, Iraq
Corresponding Author: Dr. Basma Jasims Obaid Central Teaching Hospital of Pediatric, Baghdad, Iraq

\section{Acute group a streptococcal tonsillopharyngitis in children}

\author{
Dr. Basma Jasims Obaid, Dr. Iman Abdul Wahhab Hasan and Dr. \\ Sarab Shakir Abbas
}

DOI: https://doi.org/10.33545/26643685.2019.v2.i1a.25

\begin{abstract}
Hundred patients with acute tonsillopharyngitis attending the outpatient of Central Teaching Hospital of Pediatric were studied between August 2018 and February 2019. GAS were isolated from 34 (34\%) of cases, the peak incidence was found in children between the age 6-8 years. There were no sex differences. The commonest presenting symptoms were fever, dysphagia and sore throat and the commonest signs were tonsillopharyngeal erythema, tonsillopharyngeal exudate and tender-anterior cervical lymphadenopathy. No growth or growth of normal flora found in $56(56 \%)$ of cases and other bacterial in $10(10 \%)$. Patients treated for 10 days course of oral penicillin therapy (penicillin $\mathrm{V}$ ) and the clinical cure was $22(88 \%)$ and treatment failure was found in $3(12 \%)$ of cases. No complications were encountered.
\end{abstract}

Keywords: Tonsillopharyngitis, complication, penicillin

\section{Introduction}

\subsection{Acute Pharyngitis}

It refers to any of the numerous inflammatory conditions involving the pharynx. The term is applied to conditions in which principle involvement is of throat. Tonsillitis and pharyngotonsillitis are covered under this caption ${ }^{[1,2]}$.

\subsection{Etiology}

A. Viral: Acute tonsillopharyngitis is generally caused by viral agents like Adeno viruses, Entero viruses, Herpes viruses, Epstein-Barr virus and Influenza viruses ${ }^{[3-5]}$

B. Bacterial: Group A beta-hemolytic streptococcus (GAS) is the only common bacterial causative agent (4). Less common other bacterial agents encountered are non-group A betahemolytic streptococci particularly group $\mathrm{C}$ and $\mathrm{G}^{[6-8]}$. Neisseria gonorrhoea, Corynebacterium diphtheria, Corynebacterium hemolytium, Mycoplasma and Haemophilus influenza type $b^{[3,4]}$. Neisseria gonorrhoea pharyngitis is one of the several Corynebacterium diphtheriae, extra intestinal manifestation of gonorrhea and many mimic streptococcal pharyngitis or run a subacute course and should be considered in sexually active adolescents or in young children who may have been misused ${ }^{[3,4]}$. Corynebacterium diphtheriae, although infrequent outbreak of diphtheria are likely to occur among unimmunized, low socioeconomic groups with pharyngeal pseudomembrane ${ }^{[4,9]}$. Haemophilus influenza type $b$ pharyngitis is a poorly recognized manifestation of Haemophilus influenzae infection.

1.3 Bacteriology of Beta - hemolytic streptococci: Streptococci are gram-positive cocci that grow in pairs or in variable length chain, classified on the basis of their ability to hemolyze the red blood cells: those with hemolysins producing complete hemolysis (B hemolytic), those producing partial hemolysis (a-hemolytic) and those. producing no hemolysis (y-hemolytic) ${ }^{[4,7]}$. Lancefield further separated the streptococci on the basis of difference in carbohydrate components (C-carbohydrate) within the cell wall. Betahemolysis streptococci are derided into groups A through D. Group A streptococci is one of the important groups. GAS may be differentiated from other groups by inhibition of it's growth on agar by a bacitracin disk; less than $7.5 \%$ of the other groups ("Non-group A") show such bacitracin sensitivity ${ }^{[4]}$. The outer protein of cell wall of GAS contains several antigenic proteins, the most is M-protein and hence it can be divided on the basis of their Mprotein into 80 distinct types ${ }^{[9]}$. 
Virulence and likelihood of antibody response are dependent on the presence and amount 1 of the M-protein. M-protein inhabits the phagocytosis of GAS by polymorph neutrophils ${ }^{[10]}$

Growth: Hemolytic streptococci grow poorly on ordinary nutrient agar, but. more readily on blood agar; the zones of B-hemolysis are fully developed after 18 hours growth. The colonies do not exceed $1 \mathrm{~mm}$ in size and the zone of hemolysis enlarge very little after the first 18 hours ${ }^{[11]}$.

\subsection{Acute Streptococcal tonsillopharyngitis}

1.4.1 Epidemiology: Age related attack rale: All GAS infection have the highest incidence in children younger than 10 year ${ }^{[10]}$. The peak incidence occurs in children between the ages of 5 and 15 year ${ }^{[7,1]}$ Age is not the only factor because crowded conditions in temperate climates during the winter months are associated with epidemics of pharyngitis ${ }^{[7,10,12]}$.

Sex related factor: There is no sex or race predilection ${ }^{[1]}$. Transmission of Group A Streptococcus: Person to person spread via I aerosolised microdroplets [7, 10]. Other routes including food-born out break have been well described ${ }^{[7}$, 13]. The clinical manifestation of food-borne GAS pharyngitis is more severe and more confined to the pharynx compared to the endemic air-borne disease ${ }^{[14]}$. Generally, incidence is lowest among infants who may be protected by transplacental acquisition of type-specific antibodies and lack pharyngeal receptors for streptococcal binding ${ }^{[7]}$.

- GAS are normal inhabitants of the oropharynx; colonization rates in children vary from $(15 \%-20 \%){ }^{[7}$, 10, 12]. Acquisition from an infected $\backslash$ individual is more common during the acute illness (3-5 days) and decreases during the colonization stage ${ }^{[7]}$.

\subsubsection{Clinical Features}

a. Symptoms may vary widely from very mild to severe. In older

Children there is an abrupt onset of fever and sore throat

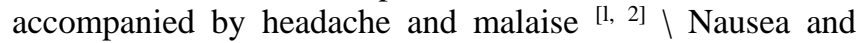
vomiting are somewhat more common in streptococcal than in non streptococcal pharyngitis, but appear to have limited usefulness as clinical prediction of streptococcal pharyngitis ${ }^{[15]}$. Younger children may present with nausea vomiting and abdominal pain.

\section{b. Physical Signs}

1. Temperature higher than $38.4{ }^{\circ} \mathrm{C}$.

2. Tonsillar enlargement with exudate.

3. Edema erythema and lymphoid hyperplasia of the pharynx.

4. Tender anterior cervical lymph nodes and petechiae on the soft palate ' History and clinical examination failed to distinguish between $3 \mathrm{AS}$ pharyngitis and pharyngitis caused by other pathogens ${ }^{[10,17]}$.

1.4.3 Investigations: Clinical diagnosis may be confirmed by culture of the throat swab or by rapid detection method for streptococcal antigen ${ }^{[2]}$.

A. Throat culture: Cultures taken with cotton swab are plated on commercially available $5 \%$ sheep's blood agar. The plate is incubated at $\left(36^{\circ} \mathrm{C}\right)$ for 24 hours and observed for characteristic white-gray colonies $1 \mathrm{~mm}$ in diameter and surrounded by a broad clear zone of hemolysis. Recovery of GAS from the throat does not necessary indicate that is the cause of the infection ${ }^{[4,9]}$. Other bacteria may proliferate during acute viral infection and may therefore be cultured in large numbers from e pharynx of an affected person but are not usually the causative agent of symptoms [3] Culture should be read after 24 horn s and if negative they should incubated from an additional 24 hours to achieve optimal sensitivity ${ }^{[5]}$.

B. Antigen detection test: A number of commercial test are available, these tests vary in method, and because the currently available tests lack sensitivity, a standard culture should be done on specimens testing negative ${ }^{[4-9]}$. One in five patients with a positive throat culture will have a negative rapid antigen test ${ }^{[8]}$. Positive results may reflect chronic colonization of GAS the acute illness is caused by another agent ${ }^{[5]}$. Antigen detection test should not be substituted for office cultures in the diagnosis of group A streptococcal throat infection ${ }^{[18]}$.

C. Streptococcal antibody test: The immunological response of the host after exposure to a streptococcal antigen can be assessed by measuring ASO and anti DNAase B (ADB) titer. BHS elaborate number of extracellular factors including pyrogenic toxin, streptolysin $\mathrm{O}$, streptolysin $\mathrm{S}$, streptokinase, hyaluronidase, proteinase, amylase and estrerase ${ }^{[7]}$. Antistreptolysin O (ASO) titers rise about 150 units within two weeks after an acute infection. Elevated ASO and anti-DNase B titers are useful in documenting prior throat infection in cases of acute rheumatic fever ${ }^{[9]}$. Streptococcal antibody test is of no immediate value in the diagnosis and treatment of acute streptococcal tonsillopharyngitis ${ }^{[5]}$.

\subsubsection{Differential diagnosis}

\section{A. Viruses}

Viruses that cause infections that mimic streptococcal sore throat acute Epstein-barr virus, Adenovirus, Herpes simplex virus, Entero Influenza virus and parainfluenza virus ${ }^{(1)}$ The pharyngitis in Herpes simplex viruses causes ulcerative lesions which most commonly involve the anterior pharynx, tongue and gums. With other viruses (Coxsackie virus and Echo viruses) rash is common ${ }^{[9]}$. Pharyngitis occurs in $85 \%$ of patients with infectious mononucleosis. Approximately $33 \%$ of patient develops an exudative pharyngitis. Older children develop the more classical picture consisting high fever, adenopathy, malaise, headache, anorexia and chills. $50 \%$ of patients develop petechial on the soft palate which in combination with oxidative pharyngitis mimics streptococcal pharyngitis ${ }^{[4]}$.
B. Bacterial agents
1. Group G and C streptococci
2. Diphtheria
3. Corynebacterium hemolyticum
4. Neisseria gonorrhea ${ }^{[4,9]}$.

\section{Other causes}

Leukemia and agranulocytosis may present with pharyngitis [9].

1.4.5 Complications of acute GAS tonsillopharyngitis ${ }^{[1]}$ : GAS infections are the focus of diagnostic and therapeutic 
efforts aimed at reducing the risk of both suppurative and non suppurative complication ${ }^{[14]}$.

a. Rheumatic fever: Physician must be aware that rheumatic fever can no longer be considered a disease of the past and be prepared to diagnose and treat promptly the children with streptococcal pharyngitis ${ }^{[20]}$. The peak incidence of initial and recurrent attacks of rheumatic fever is between 5 and 15 years of age which coincides with high frequency of GAS in this age group ${ }^{[1]}$.

b. Acute glomerulonephritis: Acute nephritis can follow streptococcal infections of either skin or pharynx in contrast to rheumatic fever, which follows pharyngeal infection only. Glomerulonephritis may occur at any age including infancy. Certain M-types are strongly associated with post-streptococcal glomerulonephritis.

The incidence of acute glomerulonephritis after streptococcal infection is variable and has ranged from nil to $28 \%$ [9]. Treatment of GAS pharyngitis not prevents the occurrence of glomerulonephritiss [22].

c. Other complications Streptococcal pharyngitis may cause the following complications

1. Large chronic ulcers in the throat.

2. Sinusitis

3. Otitis media

4. Chronic ulcer in the pharynx

5. Mesenteriadenitis ${ }^{[2]}$.

Rheumatic fever remains serious global disease for children and young adults. It decline in certain countries only emphasizes the concept this disease can be controlled and eliminated. Certain serotypes of group A streptococci (e.g. $M$ types) $[1,3,5,6,24]$ are more frequently isolated from patients with acute rheumatic fever than other serotypes. However, because the serotype is unknown at the time of clinical diagnosis of streptococcal pharyngitis, all episodes of streptococcal pharyngitis should be treated accordingly ${ }^{[7]}$

1.4.6 Treatment: Streptococcal pharyngitis shows dramatic response to oral amoxycillin is good enough). Symptomatic measures include analgesic/ antipyretics/ anti-inflammatory agents, bed rest and warm saline gargles ${ }^{[2]}$ The treatment of choice for streptococcal pharyngitis is oral amoxicillin (250 $\mathrm{mg}$ ) given 3 times daily for 10 days ${ }^{[1,23,24]}$ although failure rates of up to $20 \%$ have been reported ${ }^{[25]}$. Despite uniform susceptibility of GAS to amoxicillin, failure to eradicate GAS is not uncommon in patients receiving amoxicillin for treatment of pharyngitis [26]. Treatment after 2 days of illness increases penicillin treatment success for group A beta-hemolytic streptococcal tonsillopharyngitis ${ }^{[27]}$. Blood and tissue levels of amoxicillin sufficient to kill streptococci should be maintained for at least 10 days. Non compliant patients may be given a single intramuscular of benzathine penicillin $\mathrm{G}$, which provide adequate penicillin level for 10 days ${ }^{[1,7]}$. Intramuscular benzathine penicillin $G$ is very effective in preventing GAS pharyngitis in children. It is recommended that is used every 3 weeks at least for the fullwinter seasons in children susceptible to frequent GAS pharyngitis ${ }^{[28]}$. Erythromycin is generally effective in acute GAS pharyngitis, its use especially in children, is complicated by the high rate of gastrointestinal side effect. Erythromycin is recommended for patients who are allergic to pencillin ${ }^{[29,30]}$. Approximately 5\% of GAS are resistant to erythromycin ${ }^{[31]}$. Erythromycin estolate (20-40 $\mathrm{mg} / \mathrm{kg} /$ day in 2-4 divided ' ifcses) or erythromycin succinate (40 $\mathrm{mg} / \mathrm{kg}$ in $2-4$ divided doses) is effective in treating streptococcal pharyngitisy5,, Cephalosporins such as cephalexin also may be used to treat ssreptococcal pharyngitis, but are expensive and carry a 5-10\% risk of cross-reactivity in patients who are allergic to penicillin ${ }^{[1]}$. Cephalosporins are useful especially for the treatment of recurrent streptococcal tonsillopharyngitis ${ }^{[32]}$. Tetracycline should not be used because of the high prevalence of resistant strains which was found in $21 \%$ of GAS ${ }^{[33]}$.

Sulfonamides and trimethoprim sulfamethoxazole will not eradicate GAS in patients with pharyngitis and should not be used to treat active infections ${ }^{[5]}$.

It is necessary to improve environmental conditions of the children with particular attention is needed for improvement of ventilation, overcrowding to control those disease spreading by droplet route. Including tonsillopharyngitis.

1.4.7 Follow-up throat culture: The majority of patients with GAS pharyngitis respond clinically antimicrobial therapy, and GAS are eradicated from the pharynx. Post teament throat cultures 2-7 days after completion of therapy are indicated only in patients who remain symptomatic, who develop remain symptoms or who have had rheumatic fever and are at unusually high risk for recurrence ${ }^{[5]}$.

1.4.8 Treatment failures: There are many causes that lead to the failure of the treatment, these include the following:

1. Nonadherence to physician's instructions, including taking

2. Medications as prescribed ${ }^{[34,35]}$.

3. Bacteriological failure and clinical recurrence are strongly associated with the presence of beta-lactamase activity in commensal flora ${ }^{[36]}$

4. Unrecognized carriers.

5. Development of tolerance by streptococcus pyogenes [31].

Bacteriological treatment failure (positive culture after 14 days of treatment) occur in $4.1 \%$ of the children ${ }^{[30]}$.

1.4.9 Carriers: Chronic streptococcal carriers (defined as individuals with positive throat cultures for GAS without illness or immunologic response to GAS antigens) usually do not require treatment. ${ }^{[5]}$. The carriers of GAS increased gradually from $3 \%$ during the first year to $22 \%$ by the 5 th year ${ }^{[37]}$ Because it is impossible to distinguish carriers from infected individuals, a single course of antibiotic therapy should be administered to any patient with pharyngitis and evidence of GAS by throat swab or an antigen detection test [5]. In general, the carrier state is harmless and not contagious. An attempt to eradicate the carrier state is only if the patient or another family member has frequent GAS infectio or when they have a history of rheumatic fever or glomerulonephritis ${ }^{[31]}$ One fourth of the school children with culture positive pharyngitis actually are not truly infected ${ }^{[38]}$

\section{Aims of the study}

1. To determine the percentage of GAS as a causative microorganism of acute pharyngitis in children.

2. To find out the commonest presenting symptoms and signs of acute GAS pharyngitis in children.

3. To find out the efficacy of penicillin in the treatment of 
pharyngitis in children and whether there is treatment failure or not.

\section{Materials and Methods}

One hundred patients with acute pharyngitis visiting the Central Teaching Hospital of Paediatric were studied between August2018, and February, 2019. They were 65 boys and 35 girls and their age their was 3-14 years. Children with lower respiratory tract infection and those with acute pharyngitis who were receiving antibiotics were excluded from the study. History was obtained from the family of the patients including age, L sex, residence, similar condition in the family at the time of presentation, history of vaccination, history of rheumatic fever in the family, duration of illness, dysphagia, fever, sore throat, abdominal pain, vomiting, diarrhea and rash. Each patient was examined for the presence of tonsillopharyngeal erythema tonsillopharyngeal exudate, soft palate petechiae, abdominal tenderness, scarlatiniform rash and cervical lymphadenitis "Tender and non tender", and full systematic examination was also done. A throat swab was taken from each patient using a sterile cotton swab, which then sends to the microbiology lab. Immediately where it was inoculated on blood agar, MacConky and chocolate agar, and the results were obtained after 3 days for both culture and drug sensitivity. patients were given penicillin therapy orally 500 mg twice orally for 2 days till the results of culture and sensitivity was available. Those patients who showed GAS were asked to complete a course of 10 days and they were asked also to come back after finishing the course for re- assessment, including the presence or absence of any signs and symptoms of pharyngitis and for other throat swab. Those who positive growth of other bacteria (Streptococcal pneumoniae, non-QAS) were prescribed the proper antibiotics. Those who showed so growth or growth of normal flora were asked to stop the antibiotics. ASO titer was not done because it has no value in the immediate and in treatment of acute pharyngitis. Data obtained in this study were analyzed statistically using Z-test of two proportions between GAS pharyngitis and no growth or growth of normal.

\section{Results}

GAS was isolated from thirty-four out of one hundred patients $(34 \%)$ with acute streptococcal tonsillopharyngitis, where it was found mostly in those children between the Ages of 6-8 years. As it is shown in table (1) which also shows the age distribution according to the results of throat culture.

Table 1: Distribution of throat culture results among patients with acute pharyngitis according to age.

\begin{tabular}{|c|c|c|c|c|c|c|}
\hline $\begin{array}{c}\text { Patients age } \\
\text { (years) }\end{array}$ & GAS & \multicolumn{6}{|c|}{$\begin{array}{c}\text { Throat culture results No growth or } \\
\text { growth of normal flora }\end{array}$} \\
\hline & No & \% & No & \% & No & $\%$ \\
\hline $3-5(\mathrm{n}=32)$ & 7 & 21.78 & 22 & 68.75 & 3 & 9.37 \\
\hline $6-8(\mathrm{n}=40)$ & 18 & 45.0 & 18 & 45.0 & 4 & $10 \mathrm{D}$ \\
\hline $9-11(\mathrm{n}=20)$ & 7 & 35.0 & 11 & 55.0 & 2 & 10.0 \\
\hline $12-14(\mathrm{n}=8)$ & 2 & 25.0 & 5 & 62.50 & 1 & 12.50 \\
\hline Total & 84 & $84 \%$ & 56 & $56 \%$ & 10 & $10 \%$ \\
\hline
\end{tabular}

Table 2: Sex distribution of GAS tonsillopharyngitis. Sex distribution of GAS pharyngitis is shown in table (2).

\begin{tabular}{|c|c|c|c|c|c|}
\hline Patients age & \multicolumn{2}{|c|}{ Male patients with GAS No.\% } & \multicolumn{2}{|c|}{ Female patients with GAS No. \% } & \multirow{2}{*}{$\begin{array}{c}\text { p-value } \\
\text { Not significant }\end{array}$} \\
\hline $3-14$ & 19 & 55.88 & 15 & 44.11 & \\
\hline Total 100 & 65 & & 35 & & \\
\hline
\end{tabular}

Family history of similar illness is presented in table (3).

Table 3: Family history of similar illness in patients with acute tonsillopharyngitis (Throat culture results)

\begin{tabular}{|c|c|c|c|c|c|c|c|}
\hline Family History & \multicolumn{2}{|c|}{ GAS } & \multicolumn{2}{c|}{ No growth or growth of normal flora } & \multicolumn{2}{c|}{ Other bacteria } & p-value \\
\hline & No. & $\%$ & No. & $\%$ & No. & $\%$ & \\
\hline & 12 & 35.29 & 26 & 46.42 & 3 & 30 & N.S.* \\
\hline Total (100) & 34 & 56 & & 10 & & \\
\hline * N.S. Not Significant & & & & & & \\
\hline
\end{tabular}

The comments presenting symptoms were fever in (94.11\%) of the patient followed by dysphagia in (91.17) and the other symptoms are shown in table (4) in order of frequency.

Table 4: Correlation between symptoms of acute pharyngitis and throat culture results.

\begin{tabular}{|c|c|c|c|c|c|c|c|}
\hline Patients symptoms & & GAS & No growth or growth of normal flora & \multicolumn{2}{c|}{ Other bacteria } & p-value \\
\hline & No. & $\%$ & No. & $\%$ & No. & $\%$ & \\
\hline Fever & 32 & 94.11 & 50 & 89.28 & 7 & 70 & N.S. \\
\hline Dysphagia & 31 & 91.17 & 35 & 62.50 & 3 & 30 & $<0.001$ \\
\hline Sore throat & 26 & 76.47 & 30 & 53.57 & 5 & 50 & $<0.02$ \\
\hline Coryza & 7 & 20.58 & 10 & 17.8 & 3 & 30 & N.S. \\
\hline Abdominal pain & 7 & 20.58 & 7 & 12.50 & 2 & 20 & N.S. \\
\hline Nausea and vomiting & 3 & 8.82 & & & & & N.S. \\
\hline Diarroa & & & 8 & 14.28 & & & $<0.001$ \\
\hline Total (100) & & 34 & & 56 & & 10 & \\
\hline
\end{tabular}

The commonest physical signs were tonsillopharyngeal erythema followed by tonsillopharyngeal exudate and table (5) shows other physical signs in order of frequency.

Table 5: Correlation between signs of acute pharyngitis and throat culture result

\begin{tabular}{|c|c|c|c|c|c|c|c|}
\hline Patients signs & & GAS & \multicolumn{2}{|c|}{ No growth or growth of normal flora } & \multicolumn{2}{c|}{ Other bacteria } & p-value \\
\hline & No. & $\%$ & No. & $\%$ & No. & $\%$ & \\
\hline Tonsillopharyngeal erythema & 34 & 100.00 & 56 & 100.00 & 10 & 100.00 & \\
\hline Exudate & 20 & 58.82 & 11 & 19.64 & 3 & 30.0 & $<0.001$ \\
\hline
\end{tabular}




\begin{tabular}{|c|c|c|c|c|c|c|c|}
\hline lymph node tender & 9 & 26.47 & 2 & 5.88 & 1 & 10 & $<0.02$ \\
\hline not tender & 15 & 44.11 & 13 & 23.21 & 2 & 20 & $<0.05$ \\
\hline loft palate petechiae & 10 & 29.41 & & & & & $<0.001$ \\
\hline Abdominal tenderness & 2 & 5.88 & 3 & 5.35 & & & N.S. \\
\hline
\end{tabular}

The results of culture and sensitivity with the diameter zone of in (mm) is shown in table (6).

Table 6: Results of drug sensitivity test depending on diameter of zone of inhibition in GAS patients $(n=34)$.

\begin{tabular}{|c|c|c|c|c|c|c|c|c|c|c|}
\hline Drugs & Dp* & \multicolumn{3}{|c|}{ Susceptible } & \multicolumn{3}{c|}{ Moderate susceptible } & \multicolumn{3}{c|}{ Resistant } \\
\hline & & DZI** & No. & \% & DZI & No. & \% & DZI & No. & \% \\
\hline amoxicillin & 10 & $\geq 22$ & 24 & 70.58 & $12-21$ & 6 & 17.64 & $\leq 11$ & 7 & 11,76 \\
\hline erythromycin & 15 & $\geq 23$ & 14 & 41,17 & $14-22$ & 13 & 38.23 & $\leq 13$ & 7 & 20.5 \\
\hline cephalosporin & 30 & $\geq 18$ & 22 & 64.7 & $15-17$ & 12 & 35.29 & $\leq 14$ & - & - \\
\hline
\end{tabular}

Dp = Disk potency (Meg)

$I^{* *}$ DZI $=$ Diameter of zone of inhibition (nun) $\mid+=$ Penicillin (international unit).

There was a shortage in antibiotic disk in the lab. so that other antibiotic were not tested. Twenty-five patients came back for follow up after completing The ten days course of oral Amoxicillin, twenty-two of them were completely clinically cured and eighteen of the latter showed negative bacteriological throat culture and the rest showed positive bacteriological culture. The remaining three patients were still having some signs and symptoms of pharyngitis and their throat culture were positive for GAS, as shown in table (7).

Table 7: Patients with GAS after 10 days treatment with oral penicillin

\begin{tabular}{|c|c|c|c|c|c|c|c|}
\hline \multicolumn{2}{|c|}{$\begin{array}{c}\text { Clinical cure } \\
\text { (asymptomatic) }\end{array}$} & \multicolumn{2}{c|}{$\begin{array}{c}\text { Bacteriological cure (-ve } \\
\text { culture) }\end{array}$} & \multicolumn{2}{c|}{$\begin{array}{c}\text { Asymptomatic (+ve } \\
\text { culture) }\end{array}$} & $\begin{array}{c}\text { Treatment failure (+ve culture } \\
\text { with symptoms) }\end{array}$ \\
\hline No. & $\%$ & No. & $\%$ & No. & $\%$ & No. & $\%$ \\
\hline 22 & 88 & 18 & 72 & 4 & 16 & 3 & 12 \\
\hline
\end{tabular}

\section{Discussion}

GAS was isolated from $34(34 \%)$ of the patients of acute tonsillopharyngitis. Stjernquist-Desatnik et al. ${ }^{[39]}$, Putto-A in $1987^{[6]}$ and Mark D Widom in their study showed that GAS was encountered in $(37 \%),(31 \%)$ and one third of their patients with tonsillopharyngitis, respectively. Firas Mohammed Jamil isolated GAS from (24\%) of his acute pharyngitis patienls ${ }^{[16]}$. The difference in the rate of isolation may be related to seasonal variation or environmental factors.

\section{Age}

Fourty-five percent of the patients (18\%) were 6-8 years old in our study; also D Widome showed that $50 \%$ of his patients were 6-8 years old ${ }^{[4]}$ Wnd Putto-A found that most of his patients were 6 years old ${ }^{[6]}$, Jxit Michell M. Cloutier found that GAS was mostly isolated in those patients who are 5-15 years old ${ }^{[1]}$ so GAS is more common in school aged and less common in pre-school children.

\section{Sex}

There was no sex difference in our patients, also Michael E. Pichochero et al. showed no significant sex difference in GAS pharyngitis ${ }^{[27]}$.

\section{Family history}

Family history of similar illness was found in 12 (35.29\%) of the II| patients but Jamil M.F. noted that $(23 \%)$ of his patients had similar illness in their family ${ }^{[16]}$ The difference in the two studies may be related to more $\mathrm{HH}$ overcrowded houses in Mosul city.

\section{Clinical manifestation}

The commonest presenting symptoms were fever in 32 (94.11\%), dysphagia in 31 (91.17\%), and sore throat in 26 (76.47\%). Mitchael E. et al. in their study found that sore throat in $(99 \%), \mathrm{H}$ fever in $(84 \%)$ and headache in $(42 \%)$ were the commonest presenting symptoms [27] Jamil M.F. also found that the commonest presenting feartures were: fever $(91 \%)$, sore throat in $(88 \%)$ and dysphagia in $(73 \%)$ in his patients(16), but Kreher-NE, et al. found that abdominal pain and vomiting, were found in $(27 \%)$ and $(14 \%)$ of their study, respectively.

Edmond-KM in their study in 1996 of streptococcal pharyngitis in $\mathrm{Hi}$ a pediatric emergency department found that tender cervical lymph node, abscent croyza and scarlatiform rash were significantly associated with I GAS pharyngitis in four years old children and above ${ }^{[40]}$. The commonest signs were tonsillopharyngeal erythema in 34 $(100 \%)$, tonsillopharyngeal exudate in $20(58 \%)$ and cervical lymph adenopathy (tender and not tender) in 15, 9 (44\% and 26.47\%), respectively where as James D. Cherry said that the commonest signs in GAS pharyngitis were tonsillopharyngeal erythema in (76-100\%), j tonsillopharyngeal exudate and petechial lesion in (5175\%). Also Jamil M.F. in his study, found that tonsillopharyngeal erythema, tonsillopharyngeal exudate with anterior cervical lymphadenopathy (tender and not tender) were the commonest signs in this patients which were present in (100\%), (20\%) and (23\% and 14\%), respectively ${ }^{[6]}$. Also Adnan Dajani claimed that the above similar signs were suggestive of GAS pharyngitis in children.

\section{Treatment and follow up}

After 10 days course of oral Amxicillin therapy, clinical cure was found in $22(88 \%)$ and bacteriological cure was found in $18(72 \%)$ of patients but $4(16 \%)$ of the patients continue to have positive throat culture, although they were asymptomatic and $3(12 \%)$ of the patients were symptomatic and culture positive. Fyllingen-G et al. in their study in 1991 found that the cure rate (a combination of bacteriological and clinical cure) was $(82.0 \%)$ in the 2 dossage oral penicillin therapy. Gopichand-I et al. in their 
study found that $(70.9 \%)$ of patients were asymptomatic, $(54.5 \%)$ had bacteriological cure and (23.6\%) were asymptomatic but still carrying the GAS after completing the course of oral penicillin therapy. Michael E Pichichero, Hoeger $\mathrm{W}$, et al found in their study with twice daily oral penicillin therapy that bacteriological success rate was (94\%), (84\%), (83\%) and (73\%), respectively ${ }^{[27]}$ Disney-FA et al. in their study in 1992 found that symptomatic clinical failure occurred in $(8 \%)$ and bacteriological failure occurred in $(11 \%)$ with oral penicillin therapy. Asymptomatic patients with positive throat culture after ten days treatment with penicillin usually not required a second course of therapy which is indicated only in those with previous rheumatic fever or members of their families.

\section{Treatment failure}

Treatment failure could be explained by the following

1. A-hemolytic streptococcus is an important microorganism of normal oropharyngeal flora by inhibition of colonization and growth of GAS, but sometimes (for unknown reason) their number will decrease which flare up infection by ${ }^{\mathrm{G}} \mathrm{AS}{ }^{[25]}$.

2. The presence of beta-lactamase producing aerobic and anaerobic organisms may contribute to penicillin treatment failure by producing beta-lactamase within the tonsillar tissue and they destroy amxicillin and protect streptococci from amxicillin ${ }^{[25]}$.

3. Unrecognized carrier ${ }^{[3]}$.

4. Development of tolerance by streptococcus pyogenes. Tolerant strains demonstrate a slower rate of growth and a slower rate of bacterial killing by Amoxicillin Reinfection with the same or other serotype of GAS ${ }^{[3]}$.

\section{Conclusions}

GAS is an identifiable microorganism in causing acute pharyngitis and forming about (34\%) of all the cases.

1. The commonest age incidence was between 6-8 years.

2. There was no sex difference.

3. The commonest presenting symptoms were fever, dysphagia and sore throat and the commonest signs were tonsillopharyngeal erythema, tonsillopharyngeal exudate, and tender cervical lymphadenopathy.

4. Throat culture showed no growth or growth of normal flora in $(56 \%)$ of the cases and other bacteria (Streptococcus pneumonia, streptococcus viridance) in $(10 \%)$.

5. Oral Amoxicillin therapy for 10 days was clinically effective in $22(88 \%)$ of patients and treatment failure was found in $3(12 \%)$.

6. No complication was encountered.

\section{Recommendations}

1. Encourage parents to seek medical advice once their child suffer from fever and sore throat in order to have proper throat swab and to be given the proper antibiotic in order to prevent any complication.

2. Advice parents strongly to give the full ten days course of Amoxicillin and not stop it when they see their child asymptomatic after few days.

3. Advice the general practionals and those physicians in the primary health care center not to prescribe penicillin haphazardly to any febrile child and to send any suspicious child of having sore throat and fever for culture and sensitivity in order not to lose the efficacy of Amoxicillin by encouraging resistant microorganism.

4. Many and different antibiotic disc should be available in the laboratory of every pediatric hospital, so that better and more informative results can be obtain.

\section{References}

1. Krause P, Feder H, Greber M. Streptococal pharyngitis, Paul U, Dworking, MD. Pediatrics (3rd ed.). Awavely Company, 1996, 287-289.

2. Suraj Gupte MD. Acute pharyngitis. The Short Text Book of Pediatrics (6th ed.). Jaypee, 1986, 135-136.

3. Neile Herendeen, Peter G Szilagy. Acute pharyngitis. Nelson Text Book of Pediatrics (6th ed.). WB Saunders Co., 2000; 1264:4267. Mark D Widome Pharyngitis and tonsillitis. Hockelman RA, Blotman, Friedman, et al. Primary Pediatric Care. Crumosby Company, 1987, 1429-1433.

4. Dajani A, Taubert K, Ferrieri $\mathrm{P}$ et al. Treatment of acute streptococcal pharyngitis and prevention of rheumatic fever: a statement for health professionals. Pediatrics, 1995; 96: 29-35. Putto A. Febril Exudative Tonsillitis: Viral or streptococcal pharyngitis ? Pediatrics. 1987; 8:6-12.

5. James K Todd. Group A streptococcus. Nelson Text Book of Pediatrics (16th ed.). W.B. Saunders Company. 2000, 802-805.

6. Stromberg A, Schwan A. Department of Infectious Disease Excerpta Medica Oto Rhino-Laryngology, 1988, 481.

7. John W, Olge MD. Group A streptococcal infection. Hay, Groothuis, Hay Ward. Current Pediatric Diagnosis and Treatment (13th ed.). Appleton and Lange A, 1997, 1003-1006.

8. Denisl. Stevens, Streptococcal infection. Cecil Textbook of Medicine 1 (20th ed.). W.B. Saunders Co, 1996, 1585-1590.

9. Christie AB. Streptococcal infection. Infectious Disease (4th Ed.). Churchill Livingstone, 1987, 1276.

10. Waller WH, Detels R, Knox G. Investigative methods in public health. Oxford Textbook of public Heath, Oxford University Press, GT Britain. 1987; 3:298.

11. Michael R, Wessels. Streptococcal and enterococcal infections. Harrison's Principles of Internal Medicine 1 (14th Ed.). McGraw-Hill Book Company, 1998, 885887.

12. Bar Dayan Y, Shemer J. Food borne and air borne streptococcal pharyngitis a clinical comparison. Infection. 1997; 25:12-5.

13. Kreher NE, Hickne JM, Barry HC et al. Do gastrointestinal symptoms accompanying sore throat predict streptococcal pharyngitis ? J Fam Pract. 1998; 96:159-64

14. Jamil FM. Group A streptococcal pharyngitis among children 3-14 years of age. A thesis submitted to the Iraqi Commission for Medical Specialization in partial fulfillment of the requirement for the degree of fellowship of the Iraqi Commission for Medical Specialization in Pediatrics. University Hospital of Saddam's College of Medicine, 1997.

15. Cody DT, Kem FB, Pearson BW. Diseases of the ear, nose, throat, A Guide of Diagnosis and Management, USA, Mayo Foundation, 1981, 343-345.

16. Hoffmann S, Flenrichsen J. Detection of Group A Streptococcal antigen from throat swab by use of a 
latex agglutination test kit in general practice. Acta Pathol Immunol Scand B. 1987; 95:89-94.

17. Mandel JH. Pharyngeal infections Causes, Findings and Management. Postgrad Med. 1985; 77:187-93, 196-9.

18. McCracken GH Jr. Diagnosis and Management of children with streptococcal pharyngitis. Pediatr Infec Dis. 1986; 5:754-9.

19. Majeed HA, Yousif AM. Research on cardiac and renal sequele of streptococcal infection organized by Kuwait Heart Foundation and University of Kuwait, printed in Germany by Dustri, 1984, 128-133.

20. Alice Prince. Group A streptococcal infection. Richared E. Behrman, MD. Nelson Essential of Paediatrics (3rd ed.) W.B. Saunders Company, 1998, 373-375.

21. Shulman ST. Evaluation of penicillin, cephalosporins and macrolides for therapy of streptococcal pharyngitis. Pediatrics. 1996; 97:955-9.

22. Kunfhold A, Podlieleki A, Kuhnemund $\mathrm{O}$ et al. Infection by streptococcus pyogenes: new aspects of diagnosis, epidemiology, clinical practice, and therapy. Iinmun Infect. 1992; 20:192-9.

23. Mihajlovic Ukropina M, Roncevic N. Significance of normal oropharyngeal flora in the development of streptococcal pharyngitis and outcome of penicillin therapy. Med Pregl. 1998; 51:275-8.

24. Kim KS, Kapian EL. Association of penicillin tolerance with failure to eradicate Group a streptococcal from patients with pharyngitis. J Pdiatr. 1985; 107:681-4.

25. Michael E, Pichichero, Hoeger $\mathrm{W}$ et al. Variables influencing penicillin treatment outcome in streptococcal tonsillopharyngitis. Arch. Pediatr Adolesc Med. 1999; 153:31-36.

26. Aksit S, Caglayan S, Dokucu G. Seasonal benzathine penicillin $G$ prophylaxis for recurrent streptococcal pharyngitis in children. Acta Pediatr Jpn. 1998; 40:2568.

27. Tarlow MJ. Macrolides in the management of streptococcal pharyngitis/ tonsillitis. Pediatr Infect Dis J. 1997; 16:444-8.

28. Kiselica D. Group A beta haemolytic streptococcal pharyngitis: Current clinical concepts. Am FAM Physician. 1994; 49:1147-54.

29. RB Abrams DDS, Mueller DMD WA. Acute Streptococeal pharyngitis and tonsillitis. Hay, Groothuis, Hay Ward. Current Pediatric Diagnosis and Treatment (13th ed.). Appleton and Lange A, 1997, 421-422.

30. Scaglione F, Demartini G, Arcidicano MM et al. Optimum treatment of streptococcal pharyngitis. Drugs. 1997; 53:86-97.

31. Hoffmann S. Acta Patho Microbiol Immuno Scand B. 1985; 93:34751.

32. Sharief HJ, Tonsillitis in school children in Mosul, DIP Dissertation, University of Mosul, 1996.

33. Dajani AS. Adherence to Physicians instructions as a factor in managing streptococcal pharyngitis. Pediatrics, 1996; 97:976-80.

34. Dykhuizen RS, Golder D, Reid TM et al. Phenoxymethyl penicillin versus co-amoxiclav in the treatment of acute streptococcal pharyngitis, and the role of beta-lactamase activity in saliva. J Antimicrob Chemother. 1996; 37:133-8.

35. Amir J, Schechter Y, Eilam N. Group A beta hemolytic streptococcal pharyngitis in children younger than 5 years. J Med. Sci. 1994; 30:619-22.

36. Begovae J, Bobinac E, Benic B et al. Asymptomatic pharyngeal carriage of beta-hemolytic streptococcal and streptococcal pharyngitis among patients at an urban hospital in Croatia. Eur J Epidemiol. 1993; 9:405-10.

37. Stjernquist D, Preliner K, Christensen P. Clinical and laboratory findings in patients with acute tonsillitis. Acta Otolaryngol Stockh. 1987; 104:351-9.

38. Edmond KM, Grimwood K, Carlin JB et al. Streptococcal pharyngitis in a pediatric emergency department. Med J Aust. 1996; 165:420-3.

39. Fyllingen $\mathrm{G}$, Arnesen $\mathrm{AR}$, Biermann $\mathrm{C}$ et al. Phenoxymethylpenicillin two or three times daily for tonsillitis with beta-hemolytic streptococi group A: a blinded, randomized and controlled clinical study. Scand J Infect Dis. 1991; 23:553-8.

40. Gopichand I, Williams GD, Medendrop SV et al. Randomized single-blinded comparative study of the efficacy of amoxicillin ( $40 \mathrm{mg} / \mathrm{kg} /$ day) versus standarddose penicillin $\mathrm{V}$ in the treatment of group $\mathrm{A}$ streptococcal pharyngitis in children. Clin Pediatr. 1998; 37:341-6. 